\title{
Guest Editorial: Theme issue on location and context-aware services
}

\author{
Hui-huang Hsu • Wenny Rahayu
}

Published online: 5 March 2013

(c) Springer-Verlag London 2013

Mobile devices, especially smartphones and tablets, are becoming very popular. With the mobile technologies, it is convenient to collect and process data and to provide needed services in real time. Nowadays, people are accustomed to using a smartphone or a tablet to handle the matters in daily life. For example, online payment, personal financial management, social interaction, etc. Besides these well-accepted applications, we believe that services based on location data and context information will play a more important role in the near future. Thus, this theme issue aimed at attracting original and significant research on systems and services that sense, filter, and utilize the location of contextual information on mobile devices.

Authors with relevant papers from the AINA-2012 conference (the 26th IEEE International Conference on Advanced Information Networking and Applications) were invited to extend their papers for this theme issue. In addition, an open call for papers was also announced and circulated. At the end of the review process, we accepted seven papers for this theme issue. Each of the papers was peer reviewed by at least two experts in the field. The seven selected papers cover state-of-the-art research issues of location and context-aware services. In the following, we briefly introduce these papers.

The first paper titled "Effects of Population Size for Location-aware Node Placement in WMNs: Evaluation by a Genetic Algorithm Based Approach" by Oda et al. uses

\section{H. Hsu ( $\square)$}

Tamkang University, New Taipei City, Taiwan

e-mail: h_hsu@mail.tku.edu.tw

W. Rahayu

La Trobe University, Melbourne, Australia

e-mail: W.Rahayu@latrobe.edu.au the genetic algorithm in optimizing the deployment of mesh routers and mesh clients in wireless mesh networks (WMNs). WMNs can serve as an infrastructure for advanced location-aware services. To efficiently support such services and to offer QoS, the placement of mesh router nodes needs to be optimized. Such optimized mesh node distribution can keep the rate of location updates low and support location service managed in the mesh well.

The second paper titled "Hybrid Indoor and Outdoor Location Services for New-Generation Mobile Terminals" by Ficco et al. proposes a ubiquitous positioning system of mobile devices in both indoor and outdoor environments. It proposes a hybrid approach that can automatically switch among multiple positioning technologies, while the mobile device is moving. The location services combine signal strength-based fingerprinting techniques for indoor positioning with traditional GPS-based positioning for the outdoor localization. Opportunistic technology switching is performed according to a count-and-threshold mechanism.

The third paper titled "A Connectivity Index for Moving Objects in Indoor Cellular Space" by Alamri et al. focuses on indoor positioning of moving objects. The author proposes a new index structure based on the connectivity between the environment cells for moving objects. The index can effectively respond to the spatial indoor queries and enable efficient updates of the location of a moving object in indoor space. The proposed indoor tree performs very well in an empirical study.

The fourth paper titled "A Location-based Personal Task Reminder for Mobile Users" by Lin and Hung presents an electronic task reminder based on the calendar in smartphones. In many situations, tasks are only meaningful to be performed at a specific location. This task reminder is triggered by location of the mobile user, not by time as most of other reminder services. This work takes advantage 
of the ubiquity of IEEE 802.11 WLAN infrastructure as well as the GPS positioning. The location-based personal task reminder is effective in both indoor and outdoor environments.

The fifth paper titled "Multimedia Augmented Reality Guidance System for Museum Guidance" by Chen, Chang, and Huang developed an augmented reality-based guidance system used for museum guidance. It uses a computer vision approach. Images captured from hand motions and pictures on a printed guide are analyzed to find designated actions for prompt response to the user. Furthermore, the system can present contextual information of 3D models of museum exhibits and multimedia materials to the users in real time. The system performs better than other guidance systems in the same exhibition.

The sixth paper titled "Mobile Digcovery: Discovering and Interacting with the World through the Internet of Things" by Jara et al. introduces an homogeneous mechanism for global resource discovery, device access for deployed smart objects in different scenarios, and sensors and devices from end users. The system integrates the sensors already available from smart buildings and smart objects. Digcovery offers a framework to allow users to register their own sensors into a common infrastructure and access/discover the available resources. It utilizes the context-awareness, geo-location, and identification technologies available in smartphones.

The final paper titled "A Smart Assistant for ProductAwareness Shopping” by Chen, Huang, Park et al. presents a smart digital assistant for home furniture shopping using the RFID technology. The smart assistant allows a user to easily locate the product, check the detail information of the product, and provide real-time recommendations according to the interests of the user. Furthermore, this paper discusses a new shopping model implemented by the smart assistant. An application on smartphones is also provided along with the smart assistant.

We would like to thank the authors for contributing their valuable work to this theme issue. We are also indebted to the anonymous reviewers for their hard work. Their constructive feedbacks have helped the authors to further improve the quality of the manuscripts. Last but not least, we would like to express our gratitude to Prof. Peter Thomas, the Editor-in-Chief of the journal, for his great support and patient guidance. We hope that the papers in this theme issue will become useful references to researchers and practitioners in the field. 